is considered the least hepatotoxic. ${ }^{1}$ Except for sevoflurane, all other halogenated anaesthetics are metabolised by cytochrome P450 2E1 to produce trifluoroacetyl chloride which binds to hepatic proteins and elicits antibody and T-cell mediated responses. ${ }^{2}$ Hexafluoroisopropanol, which comprises $85 \%$ of the organic metabolites of sevoflurane, has a low binding affinity for liver macromolecules and is therefore rapidly converted to glucuronidates that are excreted in the urine. ${ }^{3}$ Proposed mechanisms of hepatic injury induced by sevoflurane include production of compound A, however, with use of Amsorb ${ }^{\circledR}$ Plus (Armstrong Medical, Coleraine, Northern Ireland), as in this case, its production is minimal. Compound A causes cellular damage by activating free radical metabolising enzymes, leading to severe injury on re-exposure, ${ }^{4}$ and increases in cytosolic free $\mathrm{Ca}^{2+}$ possibly leading to hepatocyte necrosis. ${ }^{5}$

An important factor to consider is the effect of repeat exposure of halogenated anaesthetics. The acyl metabolites of the volatile anaesthetics covalently bind to hepatic proteins and can serve as neoantigens, which may lead to sensitisation and idiosyncratic hepatotoxicity upon re-exposure to volatile anaesthetics. ${ }^{6}$ Our patient had two recent surgeries and was exposed to sevoflurane anaesthesia with both. About $95 \%$ of patients with anaesthetic-induced hepatitis have a history of fluorinated anaesthetic exposure, which is considered as a major risk factor. ${ }^{6}$

Our patient had been taking paracetamol $2.6 \mathrm{~g} \mathrm{day}^{-1}$ for 5-6 months. While the dose was $<4 \mathrm{~g}$ day $^{-1}$, considered the safe upper limit, there have been case reports where hepatic dysfunction is reported at much lower doses. ${ }^{7}$ The mechanism for this dysfunction is speculative and could be because of depleted stores of glutathione with susceptibility to injury from paracetamol at usual therapeutic doses.

Various risk factors have been described for halogenated volatile anaesthetic-induced hepatotoxicity. These include reexposure, middle age, obesity, female sex, renal failure, history of atopy or multiple drug allergies, induction of the cytochrome P450 system, and chronic ingestion of ethanol/ paracetamol. ${ }^{8}$ Our patient was middle-aged with a history of past exposure to sevoflurane and chronic ingestion of paracetamol.

The unique aspect of this report is the nature of reproducible hepatic dysfunction after sevoflurane anaesthesia in a patient with no prior history of liver disease. While the diagnosis was made by exclusion, volatile anaesthetics should be replaced by other modes of anaesthesia for future surgeries. Our recommendations for this patient are use of regional anaesthesia when feasible, total i.v. anaesthesia with processed EEG monitoring, a medical alert bracelet in case of emergent surgery, and consideration of CYP 2E1 gene polymorphism testing.

\section{Declarations of interest}

The authors declare that they have no conflicts of interest.

\section{References}

1. Nelson PD, Ferguson CN, Jones RM. Anaesthetic organ toxicity: is it really a problem? Curr Opin Anesthesiol 1998; 11: $399-401$

2. Njoku D, Laster MJ, Gong DH, Eger EI, Reed GF, Martin JL. Biotransformation of halothane, enflurane, isoflurane, and desflurane to trifluoroacetylated liver proteins: association between protein acylation and hepatic injury. Anesth Analg 1997; 84: 173-8

3. Patel SS, Goa KL. Sevoflurane. A review of its pharmacodynamic and pharmacokinetic properties and its clinical use in general anaesthesia. Drugs 1996; 51: 658-700

4. Dikmen B, Unal Y, Pampal HK, et al. Effects of repeated desflurane and sevoflurane anesthesia on enzymatic free radical scavenger system. Mol Cell Biochem 2007; 294: 31-6

5. Turillazzi E, D’Errico S, Neri M, Riezzo I, Fineschi V. A fatal case of fulminant hepatic necrosis following sevoflurane anesthesia. Toxicol Pathol 2007; 35: 780-5

6. Martin JL. Volatile anesthetics and liver injury: a clinical update or what every anesthesiologist should know. Can J Anesth Can Anesth 2005; 52: 125-9

7. Bolesta S, Haber SL. Hepatotoxicity associated with chronic acetaminophen administration in patients without risk factors. Ann Pharmacother 2002; 36: 331-3

8. Singhal S, Gray T, Guzman G, Verma A, Anand K. Sevoflurane hepatotoxicity: a case report of sevoflurane hepatic necrosis and review of the literature. Am J Ther 2010; 17: 219-22

\title{
Nocturnal desaturation early after delivery: impact of delivery type and the beneficial effects of Fowler's position
}

\author{
Nobuko Fujita ${ }^{1}$, Stephanie D. Grabitz ${ }^{2}$, Christina H. Shin², Philip E. Hess' ${ }^{2}$, \\ Noomi Mueller ${ }^{2}$, Brian T. Bateman ${ }^{2}$, Jeffrey L. Ecker ${ }^{2}$, Osamu Takahashi ${ }^{1}$, \\ Timothy T. Houle ${ }^{2}$, Yasuko Nagasaka ${ }^{1, *}$ and Matthias Eikermann ${ }^{2}$ \\ ${ }^{1}$ Tokyo, Japan and ${ }^{2}$ Boston, USA \\ *Corresponding author. E-mail: yasukonagasaka@gmail.com
}


Table 1 Effect of delivery method and sleeping position on outcomes. Values are presented as median (inter-quartile range) unless stated otherwise. "Numbers of patients for vaginal delivery or Caesarean section, in Fowler's or supine position were 20 in each group. Oxygen desaturation index defined as the average number of times per hour of valid recording time that $\mathrm{SpO}_{2}$ drop by at least $3 \%$ for a minimum duration of $10 \mathrm{~s}$.

\begin{tabular}{|c|c|c|c|}
\hline & $\begin{array}{l}\text { Delivery method } \\
\text { Vaginal delivery }(n=40)\end{array}$ & Caesarean section $(n=40)$ & $P$-value \\
\hline Duration of desaturation $\left(\mathrm{SpO}_{2}<90 \%\right.$, min) & $0.0(0.0-0.2)$ & $1.0(0.0-7.5)$ & $<0.001$ \\
\hline Time $<\mathrm{SpO}_{2} 90 \% /$ total recorded time (\%) & $0.00(0.00-0.08)$ & $0.25(0.00-3.08)$ & $<0.001$ \\
\hline Basal $\mathrm{SpO}_{2}(\%)$ & $95.4(94.7-96.1)$ & $94.6(93.2-95.4)$ & 0.002 \\
\hline Nadir $\mathrm{SpO}_{2}(\%)$ & $91(88.5-92)$ & $86(82.5-90)$ & $<0.001$ \\
\hline \multirow[t]{2}{*}{ Oxygen desaturation index $\left(\mathrm{h}^{-1}\right)$} & $3.2(2.2-5.4)$ & $4.2(2.6-7.3)$ & 0.115 \\
\hline & $\begin{array}{l}\text { Sleeping position } \\
\text { Supine }(n=40)\end{array}$ & Fowler's $(n=40)$ & $P$-value \\
\hline Duration of desaturation $\left(\mathrm{SpO}_{2}<90 \%, \mathrm{~min}\right)$ & $0.4(0.0-6.6)$ & $0.0(0.0-0.5)$ & 0.005 \\
\hline Vaginal delivery & $0.0(0.0-0.3)^{*}$ & $0.0(0.0-0.1)^{*}$ & 0.093 \\
\hline Caesarean section & $4.0(0.6-24.7)^{*}$ & $0.2(0.0-2.2)^{*}$ & 0.008 \\
\hline Time $<\mathrm{SpO}_{2} 90 \% /$ total recorded time (\%) & $0.18(0-1.54)$ & $0.00(0-0.13)$ & 0.004 \\
\hline Vaginal delivery & $0.00(0.00-0.13)^{*}$ & $0.00(0.00-0.01)^{*}$ & 0.125 \\
\hline Caesarean section & $0.99(0.24-9.79)^{*}$ & $0.06(0.00-0.50)^{*}$ & 0.003 \\
\hline Basal SpO, (\%) & $94.7(93.4-95.4)$ & $95.2(94.5-96.1)$ & 0.020 \\
\hline Nadir $\mathrm{SpO}_{2}(\%)$ & $87(82-90)$ & $90(87-92)$ & $<0.001$ \\
\hline Oxygen desaturation index $\left(\mathrm{h}^{-1}\right)$ & $3.9(2.4-6.6)$ & $3.1(2.2-5.8)$ & 0.26 \\
\hline
\end{tabular}

Editor-Women in the postpartum period are at risk of nocturnal oxygen desaturation. We have previously reported that episodes of moderate to severe obstructive sleep apnoea (OSA) occur in $20 \%$ of women in the first postpartum night and found a significant reduction in the apnoea-hypopnoea index in postpartum women who were positioned in a $45^{\circ}$ elevated upper body position (Fowler's position) compared with the horizontal position. ${ }^{1}$ Here we report a prospective, randomised, parallel-group study in postpartum women evaluating the impact of delivery type on nocturnal oxygenation and whether or not this effect is modified by elevated upper body position.

After approval of the study protocol by the Institutional Review Board (Massachusetts General Hospital, Harvard Medical School, Boston, MA, USA, Protocol \#2011P001326) and registration at clinicaltrials.gov (NCT02330055), 99 patients were stratified by delivery type and randomised on the first postpartum night to either the $45^{\circ}$ elevated (Fowler's position) or horizontal position. The primary outcome was duration of nocturnal desaturation defined as peripheral capillary oxygen saturation $\left(\mathrm{SpO}_{2}\right)<90 \%$ as detected by overnight pulse oximetry. The secondary outcomes were oxygen desaturation index, nadir $\mathrm{SpO}_{2}$, basal $\mathrm{SpO}_{2}$, and HR. Overnight pulse oximetry was conducted using a non-invasive wrist pulse oximeter (WristOxModel 3150, Nonin, Plymouth, MN, USA). Study patients were instructed to secure the pulse oximeter on to their wrist with the saturation probe on one of their fingers just before sleeping and to adjust their bed to the assigned upper body position. Obstetric nurses ensured that the patients slept in the assigned bed position throughout the study period. Data were analysed using the software nVISION (Nonin). A blinded assessor evaluated the pattern of desaturation events and manually excluded artifacts if necessary. The Wilcoxon rank sum test was used to test our primary hypothesis that postpartum women after Caesarean section (CS) are at a higher risk of desaturation than women after vaginal delivery (VD). We calculated the Hodges-Lehmann median (95\% confidence interval [CI]) differences of the duration of desaturation according to delivery mode and body position.

Of 80 patients completing the study, 40 had VD and 40 had CS with 20 patients in each delivery mode randomised to each upper body position. Time spent at $<90 \% \mathrm{SpO}_{2}$ after VD was shorter than after CS, resulting in a median (95\% CI) difference of $-0.8(-2.2$ to -0.2$)$ min $(P<0.001)$ (Table 1$)$. Duration of $\mathrm{SpO}_{2}<90 \%$ as a proportion (\%) of the total recorded time after VD was also shorter than after CS (median [95\% CI] difference of -0.22 [ -0.60 to -0.04$] \% ; P<0.001$; Table 1 ). We also found a higher incidence of severe desaturation (cumulative time of $\geq 3$ min with $\mathrm{SpO}_{2}<90 \%$ during the study night) after CS compared with VD ( $35 \%$ us $5 \%$; $P=0.001)$.

Fowler's position was associated with a shorter duration of $\mathrm{SpO}_{2}<90 \%$ compared with the horizontal position $(n=40$ per group; median [95\% CI] difference: $-0.3[-1.3$ to 0$] \mathrm{min}$, $P=0.005$; Table 1]. Also, patients in Fowler's position demonstrated a significantly lower duration of $\mathrm{SpO}_{2}<90 \%$ as a proportion of the total recorded time compared with the horizontal position (median [95\% CI] difference of -0.09 [ -0.26 to 0$] \% ; P=0.004$ ). The incidence of severe desaturation among patients in the horizontal sleeping position was higher compared with Fowler's position ( $30 \%$ vs $10 \% ; P=0.025)$. The $a$ priori subgroup analyses, stratified by delivery mode, revealed that Fowler's position significantly reduced the duratin of significant desaturation among women after CS, as compared with supine position $(P=0.009$; Table 1$)$.

We found that women in the early postpartum period are at risk of hypoxaemia during sleep. The mode of delivery was a major contributor, with VD presenting less desaturation during the 1st night after delivery. Several factors may contribute to postoperative nocturnal desaturation in patients after CS include increased oxygen consumption, pulmonary shunt because of atelectasis, and impaired upper airway patency. ${ }^{2}$ Although the median difference in the duration of nocturnal oxygen desaturation in the VD compared with the CS group 
was relatively small, it should be noted that $35 \%$ of women who had CS experienced severe desaturation us $5 \%$ of women who had VD.

Independent of the delivery mode, sleeping in Fowler's position improved oxygenation during the study night, and similarly the median difference in duration of oxygen desaturation between the Fowler's and horizontal groups was small. However, $30 \%$ of patients who slept in the horizontal position experienced severe desaturation us $10 \%$ in Fowler's position. Three mechanisms likely contribute to the beneficial effects of Fowler's position on improved upper airway patency and oxygenation in the early postpartum period: 1) gravity increases the cross-sectional area of the upper airway in postpartum women ${ }^{1}$ and improves patency of the upper airway by reducing its closing pressure and increasing stability of the upper airway ${ }^{3}$; 2) Fowler's position improves respiratory function by increasing the end-expiratory lung volume and functional residual capacity by lowering the diaphragm. Caudal position of the diaphragm acts as an oxygen reservoir and has important effects on upper airway size and resistance ${ }^{2} ; 3$ ) body fluid distribution is altered in ways that impact upper airway anatomy. ${ }^{4}$

There are several limitations to this study. First, the supine position predisposes patients with OSA to more obstructive events in the general population and thus the lateral position may benefit patients at risk of desaturation, ${ }^{5}$ however, the duration of lateral us supine in the non-elevated head position was not recorded. Second, although fluid administration is considered as an important variable that could impact the positional effect on nocturnal desaturation, this data was not accurately recorded and therefore not included in our analysis. Finally, the exact prevalence of pregnancy-associated OSA is unknown in this cohort because of the lack of polysomnography measurements.

In conclusion, CS compared with VD increases nocturnal desaturation on the 1st postpartum night, which was mitigated by Fowler's position. Further study with larger group sizes is warranted to elucidate the mechanism of the beneficial effects of Fowler's position in the early postpartum period.

\section{Authors' contributions}

Study concept and design: YN, ME.

Acquisition, analysis, and interpretation of data: SDG, CHS, PEH, NM, BTB, JLE, OT, YN, ME.

Statistical analysis: NF, SDG, CHS, YN, OT, TTH.

Drafting of the manuscript: NF, SDG, YN, ME.
Critical revision of the manuscript: NF, CHS, PEH, NM, BTB, JLE, OT, TTH,YN, ME.

Final approval of the manuscript: all authors.

Equally contributed to this study: YN, ME, NF, SDG, CHS.

\section{Declarations of interest}

TTH has received a research grant from Merck Inc, has served as a statistical consultant for Depomed Inc, receives payment for statistical reviewing from Anesthesiology, Annals of Surgery and Headache, and has received funding for research projects from National Institutes of Health (NINDS, NIGMS). ME has received funding for reserch projects from Merck, has an equity stake at Calabash Bioscience Inc, and received funding from a reserch grant from the Buzen Fund, established by Jeffrey Buzen and Judith Buzen of Boston, MA, USA. Further, he has received honoraria from Anesthesiology for editorial services. All other authors declare that they have no conflicts of interest.

\section{Funding}

Department of Anesthesia, Critical Care, and Pain Medicine at Massachusetts General Hospital, Boston, MA, USA.

\section{References}

1. Zaremba S, Mueller N, Heisig AM, et al. Elevated upper body position improves pregnancy-related OSA without impairing sleep quality or sleep architecture early after delivery. Chest 2015; 148: 936-44

2. Sasaki N, Meyer MJ, Eikermann M. Postoperative respiratory muscle dysfunction: pathophysiology and preventive strategies. Anesthesiology 2013; 118: 961-78

3. Neill AM, Angus SM, Sajkov D, McEvoy RD. Effects of sleep posture on upper airway stability in patients with obstructive sleep apnea. Am J Respir Crit Care Med 1997; 155: 199-204

4. Maw GJ, Mackenzie IL, Taylor NA. Redistribution of body fluids during postural manipulations. Acta Physiol Scand 1995; 155: 157-63

5. Jokic R, Klimaszewski A, Crossley M, Sridhar G, Fitzpatrick MF. Positional treatment vs continuous positive airway pressure in patients with positional obstructive sleep apnea syndrome. Chest 1999; 115: 771-81

\title{
Reducing the risk of confirmation bias in unrecognised oesophageal intubation
}

\author{
Daniel Jafferji, Rachael Morris and Nicholas Levy \\ Suffolk, UK \\ *Corresponding author. E-mail: Nicholas.levy@wsh.nhs.uk
}

